It is well known that pancreatic cancer is hard to cure and is often fatal. Since it presents no characteristic symptoms, and there is no effective medical check-up for the disease, it is difficult to diagnose it at an early stage. Thus how to detect a small pancreatic cancer has been a long standing question. With the rapid advancement in less invasive imaging diagnostic methods such as CT and MRI, it has become fairly easy in recent years to indirectly find abnormalities such as dilatation of the pancreatic duct, presence of pancreatic cyst, or dilatation of the bile duct. Nevertheless, there is limitation in diagnosing small pancreatic tumor by those methods.

Table 1 shows a comparison of US, CT and EUS in the ability to detect pancreatic carcinomas to be resected in our institute. Detection of tumors, particularly those smaller than $2 \mathrm{~cm}$ (TS1), was possible by EUS in most cases, except in the case of carcinoma in situ. However, detection by US was successful in $52 \%$ of the cases and by CT in $43 \%$ (Fig. 1) [4]. EUS was also helpful for detecting two different tumors located in one area (Fig. 2). From these results, it would be a key to detecting a small pancreatic cancer

\section{Small pancreatic lesions: Is there need for EUS-FNA preoperatively? What to do with the incidental lesions?}

H. Maguchi, K. Takahashi, M. Osanai, A. Katanuma

Center for Gastroenterology, Teine-Keijinkai Hospital, Sapporo, Japan

\section{Introduction}

The advantage of endoscopic ultrasonography (EUS) is its high resolution and capability of local observation [1,2]. It is one of the most accurate diagnostic methods, particularly for the pancreatobiliary region, where various advanced imaging diagnostic methods available today, such as CT and MRI $[3,4]$. Since EUS is most useful for diagnosing a small lesion, its usage for early diagnosis of pancreatobiliary cancer has being anticipated.

Endoscopic ultrasound-guided fine-needle aspiration biopsy (EUS-FNA) was developed to enhance the diagnostic capability of EUS by providing additional pathological findings [5-7].

However, it is not clearly known yet whether EUS is actually useful for the diagnosis of small pancreatic tumor, whether EUS-FNA is indispensable when a small tumor is detected. It is also necessary to understand for what size of tumor EUS-FNA can be used, and if there is no risk of complication or not. This time, I introduce the present state of diagnosing small pancreatic tumors, the application of EUS-FNA, and the discussion of the points to be solved.

Correspondence: Hiroyuki Maguchi · Center for Gastroenterology · Teine-Keijinkai Hospital · 1-jo 12-chome · Maeda · Teine-ku · Sapporo 006-8555 · Japan . Phone: 81-11-681-8111 · Fax: 81-11-685-2967 ·E-mail: maguchi@tb3.so-net. ne.jp

Bibliography: Endoscopy 2006; 38 (S1): S53-S56 (c) Georg Thieme Verlag KG Stuttgart · New York · ISSN 0013-726X · DOI 10.1055/s-2006-946653 to assemble indirect findings obtained by less invasive imaging examinations, and then resort to EUS [8].

\section{Differential diagnosis of small pancreatic lesions}

A number of reports have already shown the utility of EUS in the differential diagnosis of pancreatic tumors [9-11]. It is suitable for the diagnosis of cystic tumors, particularly the qualitative diagnosis of solid tumors presenting cystic changes (Fig. 3). Although EUS is useful in the differential diagnosis of tumors, we cannot differentiate cancerous fibrosis from inflammatory fibrosis only by the B-mode images of ultrasound. Therefore, in some cases, additional information by other modalities is required. For instance, to evaluate the vascularity of tumors is very important to differentiate pancreatic cancer from inflammatory changes. The common type of pancreatic carcinoma is usually hypovascular, in contrast to an inflammatory pancreatic mass, which is isovascular. Enhanced CT/MRI and contrast-enhanced US are useful for the differential diagnosis $[3,4,12]$. Despite the advancement in diagnostic imaging techniques, however, differentiation between pancreatic cancer and focal pancreatitis remains difficult, especially in a case of a small lesion (Fig. 4). Therefore it is sometimes required to have a tissue diagnosis. There are two methods for obtaining tissue samples from a pancreatic mass, transpapillary biopsy and/or cytology, and EUS-FNA [13-15].

Table 1 Detection rate of pancreatic carcinoma by each imaging modality

\begin{tabular}{llll} 
& US & CT & EUS \\
\hline TS1 & $11 / 21(52.4 \%)$ & $9 / 21(42.8 \%)$ & $20 / 21(95.2 \%)$ \\
\hline TS2 & $42 / 54(77.8 \%)$ & $45 / 54(83.3 \%)$ & $50 / 51(98.0 \%)$ \\
\hline TS3 & $23 / 24$ & $23 / 24$ & $20 / 20$ \\
\hline TS4 & $4 / 4$ & $4 / 4$ & $4 / 4$ \\
\hline
\end{tabular}



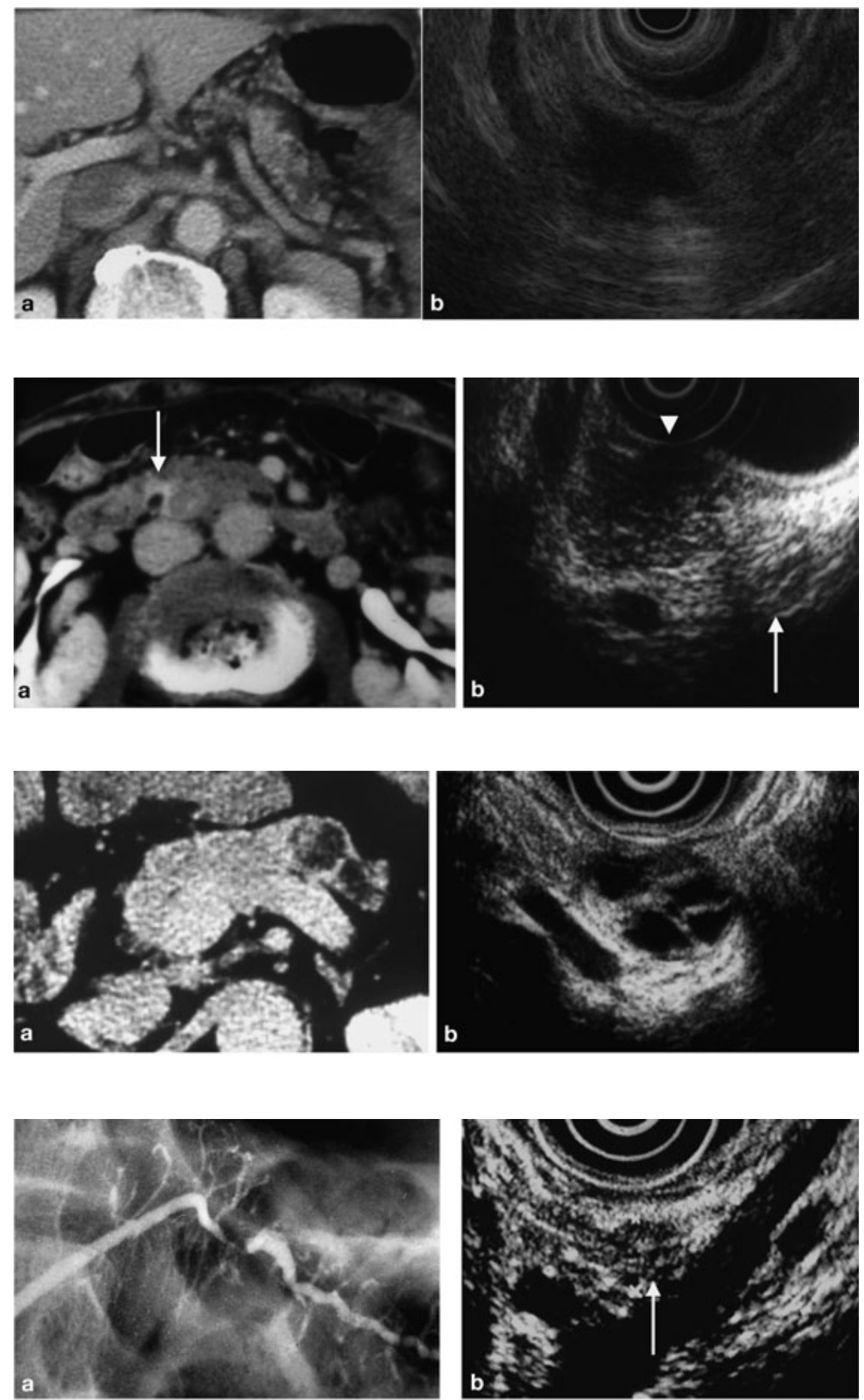

Fig. 1 A case of small pancreatic carcinoma (T1). (a) CT revealed the dilated main pancreatic duct in the pancreatic tail; however, it did not show the pancreatic tumor. (b) EUS revealed a low echoic mass.

Fig. 2 A case of pancreatic cancer concomitant with endocrine tumor. (a) CT revealed a strongly enhanced area (arrow) in the pancreatic head. (b) By EUS, two tumorous images were shown. The one close to the papilla was a homogeneous echoic mass (arrow), which was suspected to be an endocrine tumor. The other on the pancreatic body side presented an irregular periphery (arrow head), and was a hypoechoic and hetrogenious mass; it was diagnosed as pancreatic cancer.

Fig. 3 A case of endocrine tumor with cystic changes. (a) CT showed a tumor-like cystic mass in the body of the pancreas. (b) EUS visualized the internal components more precisely. This finding by EUS helped us to diagnose it as endocrine tumor with cystic changes.

Fig. 4 A case of focal pancreatits which was difficult to differentiate from small pancreatic carcinoma. (a) ERCP demonstrated a stricture of the main pancreatic duct in the pancreatic body. (b) EUS revealed a low echoic small mass measuring $6 \mathrm{~mm}$.
Transpapillary biopsy and/or cytology vs. EUS-FNA

Both methods have some advantages and disadvantages (Table 2). The advantages of transpapillary biopsy and/or cytology are being free from the risk of cancer cells dissemination and suitable for obtaining tissue samples from carcinoma in situ [16]. The disadvantages arise in the difficulty to obtain tissue samples from the branch duct of the pancreas, risk of ERCP-related pancreatitis, and that the techniques are sometimes difficult and the sensitivity is less than what we expected. In contrast, the advantages of EUSFNA are being free from the ERCP-related pancreatitis and suitable for obtaining tissue samples even if cancer has not invaded the main pancreatic duct. The disadvantages are the risk of dissemination of cancer cells after EUS-FNA and the difficulty to obtain tissue samples from the carcinoma in situ; and it is not clear yet on what size of tumor EUS-FNA can be applied to. 


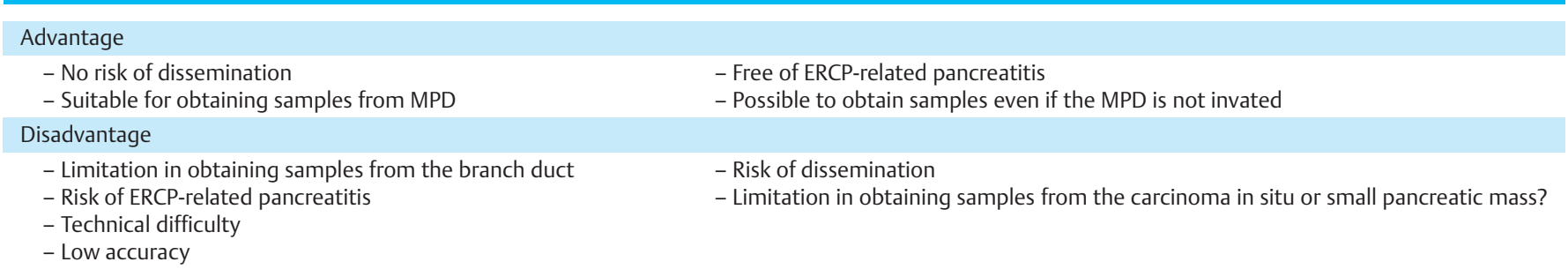

\section{Preoperative histological diagnosis of small pancreatic} carcinoma

There are very few reports of histologically diagnosed small pancreatic carcinoma preoperatively. Yamao et al. [17] reported the results of biopsies by ERCP and EUS-FNA in operated patients with small pancreatic cancer less than $2 \mathrm{~cm}$ in diameter (TS1); the cancer-positive rate was 50\%(4/8) in the use of ERCP and $100 \%(2 / 2)$ in the use of EUS-FNA. We have investigated the results of transpapillary biopsy/cytology in our operated patients with TS1; the cancer-positive rate was $56 \%(10 / 18)$. We have not performed EUS-FNA on patients with TS1 because we are very concerned with dissemination of malignant cells after EUS-FNA.

\section{Indication of EUS-FNA for small pancreatic mass lesions}

A fundamental principle in establishing indication for EUS-FNA is the determination as to whether or not the information obtained has the potential to affect patient management [18]. According to this principle, EUS-FNA is currently indicated for pancreatic mass lesions for (1) histological evidence required for chemotherapy and/or radiation therapy, (2) differential diagnosis between localized pancreatitis and pancreatic cancer which shows an atypical imaging pattern and/or is negative for biopsy/ cytology by transpapillary approach, and (3) tumor staging in a patient with a small amount of ascitis or lymph node swelling [17]. However, the role of EUS-FNA in patients suspected of having pancreatic cancer that appears to be respectable on imaging studies especially small lesion is controversial. One view is that a tissue diagnosis will not alter patient management, and is therefore unnecessary. This is because as with EUS-FNA for pancreatic masses have a low negative predictive value for malignancy. Thus, FNA negative for cancer will not exclude the suspected diagnosis of cancer and the patient will be explored anyway. In addition, the risk of tumor seeding by EUS-FNA is strongly stressed in the opinions against the indication of this approach, especially in Japan [17]. It has not fully been determined whether tumor seeding occurs with EUS-FNA. There have been three reports of seeding possibly caused by EUS-FNA [18-21]. Paquin et al. [21] reported of tumor seeding after EUS-FNA in patients with pancreatic cancer even though the tumor size was $8 \mathrm{~mm}$.

Theoretically tumor seeding may occur more frequently in cystic lesions than in solid lesions [17-19], and the complication from EUS-FNA appears to have a greater risk of infections in cystic le- sions; the incidence rate is $14 \%$ compared to $0.5 \%$ in solid masses. For this reason, indication or contraindication of EUS-FNA for pancreatic cystic lesions suspected of being neoplastic cystic tumors is a matter of controversy in Japan, which explains why EUS-FNA has actively been performed for such lesions in the United States and Europe [22,23], but not in Japan. In view of the above, it will be our future task to further the study of tumor seeding and to devise a reliable method of tissue sampling in the use of EUS-FNA.

\section{References}

${ }^{1}$ Rosch T, Braig C, Gain T et al. Staging of pancreatic and ampullary carcinoma by endoscopic ultrasonography: comparison with conventional ultrasonography, computed tomography, and angiography. J Gastroenterol 1992; 102: 188-199

2 Yasuda K, Mukai H, Nakajima M et al. Staging of pancreatic carcinoma by endoscopic ultrasonography. Endoscopy 1993; 25: 151 - 155

${ }^{3}$ Maguchi H. Roles of endoscopic and intraductal ultrasonography in the diagnosis of pancreaticobiliary lesions. Dig Endosc 2001; 13: S42 - 46

${ }^{4}$ Maguchi H. Roles of endoscopic ultrasonography in the diagnosis of pancreatic tumors. J Hepatobiliary Pancreat Surg 2004; 11: 1-3

5 Vilmann P, Jacobsen GK, Henriksen FW et al. Endoscopic ultrasonography with guided fine needle aspiration biopsy in pancreatic disease. Gastrointest Endosc 1992; 38: 172 - 173

${ }^{6}$ Tio TL, Sie LH, Tygat GN. Endosonography and cytology in diagnosing and staging pancreatic body and tail carcinoma. Preliminary results of endosonographic guided puncture. Dig Dis Sci 1993; 38: 59-64

${ }^{7}$ Binmoeller KF, Jabusch HC, Seifert $\mathrm{H}$ et al. Endosonography-guided fine-needle biopsy of indurated pancreatic lesions using an automated biopsy device. Endoscopy 1997; 29: $384-388$

${ }^{8}$ Shimizu Y, Yasui K, Matueda K et al. Small carcinoma of the pancreas is curable: new computed tomography findings, pathological study and postoperative results from a single institute. J Gastroenterol Hepatol 2005; 20: 1591 - 1594

${ }^{9}$ Muller MF, Meyenber C, Bertschinger P et al. Pancreatic tumors: evaluation with endoscopic US, CT, and MR imaging. Radiology 1994; 190 : $745-751$

${ }^{10}$ Furukawa T, Tsukamoto Y, Naitoh Y et al. Differential diagnosis of pancreatic diseases with an intraductal ultrasound system. Gastrointest Endosc 1994; 40: $213-219$

${ }^{11}$ Koito K, Namieno T, Nagakawa T et al. Solitary cystic tumor of the pancreas: EUS-pathological correlation. Gastrointest Radiol 1997; 45: $268-276$

12 Koito K, Namieno T, Nagakawa T et al. Inflammatory pancreatic masses: differentiation from ductal carcinomas with contrast-enhanced sonography using carbon dioxide microbubbles. Am J Radiology 1997; 169: $1263-1267$

${ }^{13}$ Takahashi K, Yamao K, Okubo K et al. Differential diagnosis of pancreatic cancer and focal pancreatitis by using EUS-guided FNA. Gastrointst Endosc 2005; 61: 76 - 79

${ }^{14}$ Itoi T, Itokawa F, Sofuni A et al. Pancture of solid pancreatic tumors guided by endoscopic ultrasonography: a pilot study series comparing Trucut and 19-gauge and 22-gauge aspiration needles. Endoscopy 2005; 37: $362-366$ 
${ }^{15}$ Ryozawa S, Kitoh H, Gondo T et al. Usefulness of endoscopic ultrasound-guided fine-needle aspiration biopsy for the diagnosis of pancreatic cancer. J Gastroenterol 2005; 40: 907-911

${ }^{16}$ Obara T, Maguchi H, Saitoh Y et al. Intraductal papillary neoplasms of the pancreas: diagnosis by Endoscopic Pancreatic Biopsy. Endoscopy 1993; 25: 290-293

17 Yamao K, Sawaki A, Mizuno N et al. Endoscopic ultrasound-guided fine-needle aspiration biopsy(EUS-FNAB): past, present, and future. J Gastroenterol 2005; 40: 1013-1023

${ }^{18}$ Howes RH. Indications for EUS-directed FNA. Endoscopy 1998; 30: A155- 157

${ }^{19}$ Hirooka Y, Goto H, Itoh A et al. Case of intraductal papillary mucinous tumor in which endosonography-guided fine-needle aspiration biopsy caused dissemination. J Gastroenterol Hepatol 2003; 18: 1323 1324

${ }^{20}$ Shah JN, Fraker D, Guerry D et al. Melanoma seeding of an EUS-guided fine needle track. Gastrointest Endosc 2004; 59: 923 - 924

${ }^{21}$ Paquin SC, Gariepy G, Lepanto L et al. A first report of tumor seeding because of EUS-guided FNA of a pancreatic adenocarcinoma. Gastrointest Endosc 2005; 61: 610-611

${ }^{22}$ Frossard JL, Amouyal P, Amouyal G et al. Performance of endosonography-guided fine needle aspiration and biopsy in the diagnosis of pancreatic cystic lesions. Am J Gastroenterol 2003; 98: 1516-1524

${ }^{23}$ Hernandez LV, Mishra G, Forsmark C et al. Role of endoscopic ultrasound(EUS) and EUS-guided fine needle aspiration in the diagnosis and treatment of cystic lesions of the pancreas. Pancreas 2002; 25: $222-228$ 